\title{
Neutrophil-independent mechanisms of caspase-1- and IL-18-mediated ischemic acute tubular necrosis in mice
}

\author{
Vyacheslav Y. Melnikov, ${ }^{1}$ Sarah Faubel, ${ }^{1}$ Britta Siegmund, ${ }^{1}$ M. Scott Lucia, ${ }^{2}$ \\ Danica Ljubanovic, ${ }^{2}$ and Charles L. Edelstein ${ }^{1}$ \\ ${ }^{1}$ Department of Medicine, and \\ ${ }^{2}$ Department of Pathology, University of Colorado School of Medicine, Denver, Colorado, USA
}

\begin{abstract}
Having recently described the injurious role of caspase-1-mediated production of the proinflammatory cytokine IL-18 in ischemic acute renal failure (ARF), we report here on the effect of the newly developed caspase inhibitor Quinoline-Val-Asp (Ome)- $\mathrm{CH}_{2}-\mathrm{OPH}(\mathrm{OPH}-001)$ on caspase-1, IL-18, neutrophil infiltration, and renal function in ischemic ARF. C57BL/6 mice with ischemic ARF treated with OPH-001 had a marked (100\%) reduction in blood urea nitrogen (BUN) and serum creatinine and a highly significant reduction in morphological acute tubular necrosis (ATN) score compared with vehicle-treated mice. OPH-001 significantly reduced the increase in caspase-1 activity and IL-18 and prevented neutrophil infiltration in the kidney during ischemic ARF. To evaluate whether this lack of neutrophil infiltration was contributing to the protection against ischemic ARF, a model of neutrophil depletion was developed. Neutrophil-depleted mice had a small (18\%) reduction in serum creatinine during ischemic ARF but no reduction in ATN score despite a lack of neutrophil infiltration in the kidney. Remarkably, caspase-1 activity and IL-18 were significantly increased in the kidney in neutrophil-depleted mice with ARF. In addition, IL-18 antiserum-treated neutrophil-depleted mice with ischemic ARF had a significant (75\%) reduction in serum creatinine and a significant reduction in ATN score compared with vehicletreated neutrophil-depleted mice. These results suggest a novel neutrophil-independent mechanism of IL-18-mediated ischemic ARF.
\end{abstract}

J. Clin. Invest. 110:1083-1091 (2002). doi:10.1172/JCI200215623.

\section{Introduction}

The caspases are a family of intracellular cysteine proteases. Caspases participate in two distinct signaling pathways: (a) activation of proinflammatory cytokines by caspase- 1 (previously known as IL-1 $\beta$ converting enzyme, or ICE), and (b) promotion of apoptotic cell death via caspase-3. There is now considerable evidence that caspases are also involved in necrotic cell death in vitro. Inhibition of caspases protects against necrotic cell death induced by hypoxia in renal tubules in culture (1) and freshly isolated rat proximal tubules (2). In rat kidneys with acute tubular necrosis (ATN), both caspase- 1 and caspase- 3 mRNA and protein expression (3) as well as caspase- 3 activity (4) are increased. Caspase inhibition attenuates distal tubule apoptosis and inflammation in ischemic acute renal failure (ARF) in mice

Received for publication April 5, 2002, and accepted in revised form August 20, 2002.

Address correspondence to: Charles L. Edelstein, Division of Renal Diseases and Hypertension, University of Colorado School of Medicine, Box C281, 4200 East 9th Avenue, Denver, Colorado 80262, USA. Phone: (303) 315-8764;

Fax: (303) 315-4852; E-mail: Charles.edelstein@uchsc.edu. Conflict of interest: No conflict of interest has been declared. Nonstandard abbreviations used: acute tubular necrosis (ATN); acute renal failure (ARF); Quinoline-Val-Asp (Ome)- $\mathrm{CH}_{2}-\mathrm{OPH}$ (OPH-001); blood urea nitrogen (BUN);

electrochemiluminescence (ECL); myeloperoxidase (MPO); lactate dehydrogenase (LDH); not significant (NS).
(5). However, the effect of caspase inhibitors on ATN, the predominant pathological process in animal models of ischemic ARF and in posttransplant ARF in humans, is not known. Thus, on the background of caspase inhibitor studies in vitro in proximal tubules and in vivo studies in kidney, we determined the effect of the newly developed caspase inhibitor Quinoline-Val-Asp(Ome)- $\mathrm{CH}_{2}-\mathrm{OPH}$ (OPH-001) on the functional and morphological changes in ischemic ARF in mice. While the use of caspase-deficient mice has provided extensive information about the role of individual caspases in disease processes, the study of caspase inhibitors in vivo represents an important initial step toward possible therapeutic effects of caspase inhibition.

The proinflammatory caspase- 1 plays a major role in the cleavage of the IL- $1 \beta$ precursor and the IL-18 precursor. Caspase- 1 is remarkably specific for the precursors of IL- $1 \beta$ and IL-18 (IFN- $\gamma$-inducing factor) by making a single initial cut in each procytokine, which results in an active mature cytokine secreted into the extracellular space (6). We have demonstrated that caspase-1-deficient mice are functionally and histologically protected against ischemic ARF and that this protection is associated with decreased conversion of IL-18 precursor to the mature form in the kidney (7). In this study, the administration of IL-18neutralizing antiserum protected against ischemic $\mathrm{ARF}$, confirming the deleterious role of IL-18 in the 
pathogenesis of ischemic ARF. Both caspase-1-deficient mice and mice treated with IL-18-neutralizing antiserum had decreased neutrophil infiltration in the kidney during ischemic ARF.

The role of neutrophils in the pathogenesis of ARF remains controversial. A model of neutrophil depletion in mice that uses the specific neutrophil-depleting mAb RB6-8C5 has recently been developed (8). We have reproduced this model of neutrophil depletion in ischemic ARF in mice. In the present study, we used a caspase inhibitor, IL-18-neutralizing antiserum, and neutrophil-depleted mice to test the hypotheses that caspase inhibition protects against ischemic ARF and that caspase-1-mediated production of IL-18 can induce ischemic ARF in the absence of neutrophils.

\section{Methods}

Ischemia protocol. For all the mouse studies, C57BL/ 6 mice (The Jackson Laboratory, Bar Harbor, Maine, USA) were used. Mice weighing 20-25 g were anesthetized with an intraperitoneal injection of Avertin (2,2,2-tribromoethanol; Sigma-Aldrich, Milwaukee, Wisconsin, USA). A midline incision was made, and the renal pedicles were bilaterally clamped for $22 \mathrm{~min}$ utes with microaneurysm clamps. The time of ischemia was chosen to obtain a reversible model of ischemic ARF and to avoid animal mortality. Serum creatinine reaches a peak at 24-48 hours of reperfusion and then gradually returns to normal within 3-7 days. After 22 minutes the clamps were removed. The kidneys were observed for restoration of blood flow, as demonstrated by a return to their original color. The abdomen was closed in two layers. Sham surgery consisted of the same surgical procedure except that clamps were not applied. During the first 24 hours of the reperfusion period, the animals were kept in an incubator at $29^{\circ} \mathrm{C}$. In separate experiments, blood samples were obtained via cardiac puncture at 24,48 , and 72 hours of renal reperfusion. Blood urea nitrogen (BUN) and serum creatinine were measured using a BUN and a creatinine autoanalyzer (Beckman Instruments Inc., Fullerton, California, USA).

Caspase inbibition. Quinoline-Val-Asp (Ome)- $\mathrm{CH}_{2}-$ $\mathrm{OPH}$ [Q-VD-(Ome)-OPH] was obtained from Enzyme Systems Products Inc. (Livermore, California, USA). The inhibitor $(120 \mathrm{mg} / \mathrm{kg})$ or vehicle (high-purity DMSO; Sigma-Aldrich, St. Louis, Missouri, USA) was administered intraperitoneally 60 minutes before renal pedicle clamp.

OPH-001 is a new-generation broad-spectrum caspase inhibitor. Compared with the benchmark caspase inhibitor Z-Val-Ala-Asp(Ome)- $\mathrm{CH}_{2} \mathrm{~F}$ (Z-VADFMK), it has significant differences in structural design. These changes include the replacement of the carbobenzoxy blocking group $(Z)$ with a quinoline derivative $(\mathrm{Q})$, modification of the tripeptide sequence from VAD to VD, and replacement of the putatively toxic fluoromethyl ketone (FMK) with the nontoxic 2,6-difluorophenoxy (OPH) group. The mechanism of action involves the formation of an irreversible thioether bond between the aspartic acid derivative in the inhibitor and the active site cysteine of the caspase with the displacement of the 2,6difluorophenol group.

Histological examination. Kidneys fixed in 4\% paraformaldehyde and embedded in paraffin were sectioned at $4 \mu \mathrm{m}$ and stained with hematoxylin and eosin and periodic acid-Schiff by standard methods. Histological examinations were performed by the renal pathologist in a blinded fashion. Histological changes due to tubular necrosis were quantitated by calculation of the percent of tubules that displayed cell necrosis, loss of brush border, cast formation, and tubule dilatation as follows: 0 , none; $1, \leq 10 \% ; 2$, $11-25 \% ; 3,26-45 \% ; 4,46-75 \%$; and 5, > 76\%. At least ten fields $(\times 200)$ were reviewed for each slide.

The renal pathologist quantitatively assessed neutrophil infiltration in a blinded fashion by counting the number of neutrophils per $\mathrm{mm}^{2}$ at $\times 400$ using a calibrated ocular grid. At least ten fields were counted in the outer medulla on slides stained with hematoxylin and eosin.

Morphologic criteria were used to count apoptotic cells on hematoxylin and eosin staining. These characteristics included cellular rounding and shrinkage, nuclear chromatin compaction, and formation of apoptotic bodies (9). Apoptotic tubular cells were quantitatively assessed per ten high-power fields $(x 400)$ by the renal pathologist in a blinded fashion.

Caspase activity assay. Caspase activity in the kidney was determined by use of fluorescent substrates as we have previously described (7).

The assay of purified caspases was performed as follows. Purified recombinant caspase-3 (Upstate Biotechnology Inc., Lake Placid, New York, USA) and recombinant caspase-1 purified to homogeneity from Escherichia coli (obtained from Nancy Thornberry, Merck Research Laboratories, Rahway, New Jersey, USA) were used to calculate the OPH-001 concentration that inhibits the purified caspase- 1 or -3 concentration by $50 \%\left(\mathrm{IC}_{50}\right)$. Recombinant caspase- 1 or caspase- 3 was diluted in the following buffer to convert it to the active form: $100 \mathrm{mM}$ Na HEPES, $10 \mathrm{mM}$ DTT, $1 \mathrm{mM}$ EDTA, 0.1\% 3-[(3-cholamidopropyl) dimethylammonio]-1-pro-panesulfonate (CHAPS), and $10 \%$ sucrose. Ten nanograms of the activated caspase was added to the caspase- 1 or caspase- 3 assay buffer. The caspase- 1 assay buffer contained $25 \mathrm{mM}$ $\mathrm{K}^{+}$HEPES, 1 mM DTT, 1 mM EDTA, 0.1\% CHAPS, 1 mM DTT, and $10 \%$ sucrose, $\mathrm{pH}$ 7.5. The caspase- 3 assay buffer contained $25 \mathrm{mM} \mathrm{K}{ }^{+}$HEPES, KCl 50 $\mathrm{mM}, 1 \mathrm{mM}$ DTT, 0.1\% CHAPS, and $1 \mathrm{mM}$ DTT, $\mathrm{pH}$ 7.5. Increasing concentrations of $\mathrm{OPH}-001$ were added as follows: $0 \mu \mathrm{M}$ (vehicle only), $0.1 \mu \mathrm{M}, 1 \mu \mathrm{M}$, $10 \mu \mathrm{M}, 100 \mu \mathrm{M}$, and $500 \mu \mathrm{M}$. After 10 minutes' preincubation, $10 \mu \mathrm{l}$ of the substrate (final concentration, $50 \mu \mathrm{M})$ was added to make a total volume of $200 \mu \mathrm{l}$. 
Acetyl-Tyr-Val-Ala-Asp-7-amido-4-methyl coumarin (Ac-YVAD-AMC) in 10\% DMSO was used as a substrate for caspase-1-like proteases. Acetyl-Asp-Glu-ValAsp-7-amido-4-methyl coumarin (Ac-DEVD-AMC) in $10 \%$ DMSO was used as a substrate for caspase- 3 .

Calpain and cathepsin B assay. Calpain and cathepsin B activity were determined by use of fluorescent substrates as we have previously described (4). Purified $\mu$-calpain $(2.5-5 \mu \mathrm{g})$ from porcine erythrocytes (Calbiochem-Novabiochem Corp., San Diego, California, USA) or purified cathepsin B (0.3-0.6 $\mu \mathrm{g})$ from human liver tissue (BIOMOL Research Laboratories Inc., Plymouth Meeting, Pennsylvania, USA) was used to calculate the $\mathrm{IC}_{50}$ for OPH-001. $\mathrm{N}$-succinyl-Leu-Tyr-7amido-4-methyl coumarin (Sigma-Aldrich) in DMSO was used as a substrate for calpain. Z-Phe-Arg-AMC (Peptide Institute, Osaka, Japan) in DMSO was used as a substrate for cathepsin $B$.

The cathepsin B assay in kidney was performed as follows. Briefly, kidney tissue was mixed with lysis buffer and homogenized with ten strokes in a glass-Teflon homogenizer. The lysate was then centrifuged at $4{ }^{\circ} \mathrm{C}$ at $100,000 \mathrm{~g}$ in a Beckman Instruments Inc. Ti70 rotor for 1 hour. The resultant supernatant $(100 \mu \mathrm{g}$ protein) was assayed for cathepsin B as described above.

Electrochemiluminescence assay for IL-18. The electrochemiluminescence (ECL) assay for IL-18 in whole kidney homogenates was performed as previously described in detail (10). The ECL assay detects both pro-IL-18 and mature IL-18.

Rabbit anti-murine IL-18-neutralizing antiserum. Rabbit anti-murine IL-18-neutralizing antiserum was obtained from a New Zealand rabbit immunized by intradermal injection of murine recombinant IL-18 in the presence of Hunter's titermax adjuvant (6). The IL-18 antiserum has been used in mice in vivo to block endogenous IL-18 (10). Rabbit anti-murine IL-18-neutralizing antiserum or vehicle (normal rabbit serum) was administered as follows: $300 \mu \mathrm{l}$ intraperitoneally 40 minutes before renal pedicle clamp, and $100 \mu \mathrm{l}$ intraperitoneally just before clamp release.

Neutrophil depletion model. Mice were injected with 0.1 mg of the rat IgG2b mAb RB6-8C5 (BD Pharmingen, San Jose, California, USA) intraperitoneally 24 hours before renal pedicle clamp (8). This results in depletion of neutrophils in the peripheral blood and in the kidney during ischemic ARF (see Results).

Myeloperoxidase assay. Myeloperoxidase (MPO) activity (kinetic assay) was measured as previously described, with modifications (11).

Preparation of proximal tubules. Proximal tubules were isolated from kidney cortex of male C57BL/6 mice using collagenase digestion and Percoll centrifugation (2). Six-milliliter aliquots of tubule suspension (about $1-2 \mathrm{mg} / \mathrm{ml}$ ) were placed in siliconized $25-\mathrm{ml}$ Erlenmeyer flasks. To create hypoxia, the tubule suspension was gassed in a shaking water bath with $95 \% \mathrm{~N}_{2}-5 \% \mathrm{CO}_{2}$ at a rate of $3 \mathrm{l} / \mathrm{min}$. At the end of the hypoxic period, $1 \mathrm{ml}$ of tubule suspension was sampled for measurement of lactate dehydrogenase $(\mathrm{LDH})$ and $2 \mathrm{ml}$ for immunoblotting. $\mathrm{LDH}$ release into the tubule medium was used as an index of cell membrane damage.

Western blot analysis. Kidney cortices or proximal tubules were homogenized in radioimmunoprecipitation assay (RIPA) buffer, and Western blotting was performed using standard protocols as previously described (4). A goat anti-IL-18 polyclonal antibody (Santa Cruz Biotechnology Inc., Santa Cruz, California, USA; 1:100) was used.

Statistical analysis. Non-normally distributed data were analyzed by the nonparametric unpaired MannWhitney test. Multiple group comparisons were performed using ANOVA with posttest according to Newman-Keuls. A $P$ value of less than 0.05 was considered statistically significant. Values are expressed as means \pm SE.

\section{Results}

Mice treated with OPH-001 do not develop functional ischemic ARF. Mice were treated with vehicle (DMSO) or OPH-001 $(120 \mathrm{mg} / \mathrm{kg})$ before induction of ischemic ARF. Mice treated with OPH-001 before induction of ARF had a $100 \%$ reduction in serum creatinine at 24 hours of reperfusion compared with vehicle-treated mice with ischemic ARF, and a serum creatinine level similar to that of sham-operated controls (Figure 1). At 48 hours of reperfusion, OPH-001-treated mice remained completely protected against ischemic ARF (Figure 1). By 72 hours of reperfusion, renal function returned to normal in vehicle-treated mice, confirming the reversible nature of this model of ischemic ARF (Figure 1). BUN demonstrated a similar pattern at 24 hours of reperfusion. BUN (mg/dl) was $24 \pm 0.7$ in sham-operated control mice, $102.4 \pm 13$ in mice with vehicle-treated ischemic $\operatorname{ARF}(P<0.001$ vs. sham, $n=6-8)$, and $36 \pm 4$ in OPH-001-treated mice with ischemic ARF $(P<0.01$ vs. vehicle-treated ischemic ARF, not significant [NS] vs. sham, $n=6-8)$. A dose of $120 \mathrm{mg} / \mathrm{kg} \mathrm{OPH}-001$ was chosen, as it gave the best functional protection compared with $40 \mathrm{mg} / \mathrm{kg}$ and $80 \mathrm{mg} / \mathrm{kg}$.

Sham-operated mice had an ATN score of 0, representing no tubular necrosis (Figure 2). Vehicle-treated mice with ARF had a mean ATN score of 4.7, representing necrosis in $46-75 \%$ of tubules (Figure 2). Mice treated with OPH-001 before induction of ARF had a mean ATN score of 1.8, representing tubular necrosis in 11-25\% of tubules (Figure 2). Renal histopathology is shown in Figures 3, a and b.

OPH-001 inhibits caspase-1 activity, IL-18 protein expression, and neutrophil infiltration during ischemic ARF. OPH-001 inhibited the increase in caspase- 1 activity in whole kidney homogenates during ischemic ARF (Figure 4a). IL-18 protein was determined by the ECL assay in kidney homogenates. IL-18 protein was increased in vehicle-treated mice with ischemic ARF compared with sham-operated control mice (Figure $4 \mathrm{~b}$ ). There was a 


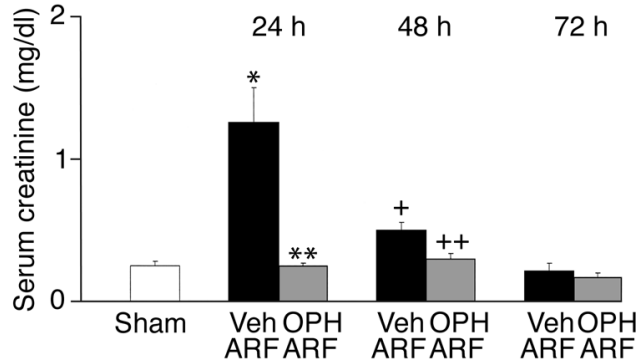

\section{Figure 1}

Mice treated with $\mathrm{OPH}-001$ are functionally protected against ischemic ARF. In vehicle-treated mice with ischemic ARF, serum creatinine was significantly increased at 24 hours and 48 hours of reperfusion compared with sham-operated controls. In mice treated with OPH-001 before induction of ischemic ARF, the serum creatinine was normalized at 24 hours and 48 hours of reperfusion compared with sham-operated controls. ${ }^{*} P<0.01 \mathrm{vs}$. sham; ${ }^{*} P<0.01$ vs. vehicle-treated ARF, NS vs. sham; $n=8$ at 24 hours. ${ }^{+} P<0.05$ vs. sham; ${ }^{++} P<0.05$ vs. vehicle-treated ARF, NS vs. sham; $n=6$ at 48 and 72 hours. Veh, vehicle.

large reduction in IL-18 protein in OPH-001-treated mice with ischemic ARF compared with vehicle-treated mice with ischemic ARF (Figure 4b).

Because OPH-001 inhibited both caspase-1 and IL-18, both of which are proinflammatory, its effect on inflammation during ischemic ARF was determined. MPO activity in ischemic ARF was decreased in OPH-001-treated mice. MPO activity (OD/min/mg) was $0.003 \pm 0.2$ in sham-operated control mice, $0.056 \pm 0.012$ in vehicle-treated mice with ischemic ARF $(P<0.5$ vs. sham, $n=4)$, and $0.018 \pm 0.005$ in OPH-001treated mice with ischemic ARF $(P<0.05$ vs. vehicletreated ischemic ARF, $n=4$ ).

Because MPO activity identifies the activity of monocytes and macrophages as well as neutrophils, neutrophil infiltration in the kidney was quantified (12). Neutrophil infiltration (neutrophils $/ \mathrm{mm}^{2}$ ) in the outer medulla was $4.3 \pm 0.9$ in sham-operated animals, $594 \pm 199$ in vehicle-treated mice with ischemic ARF $(P<0.01$ vs. sham $)$, and $10.8 \pm 4$ in $\mathrm{OPH}-001$-treated mice with ischemic ARF $(P<0.01$ vs. vehicle-treated ischemic ARF, NS vs. sham) (Figure 4c). Thus, neutrophil infiltration was increased more than 100-fold in mice with ischemic ARF, and OPH-001 completely prevented the increase in neutrophil infiltration in the kidney during ischemic ARF.

Mouse model of neutrophil depletion. Mice were injected with $0.1 \mathrm{mg}$ of the rat IgG2b mAb RB6-8C5 (BD Pharmingen) or vehicle (sterile water) intraperitoneally 24 hours before renal pedicle clamp (8). The mAb results in depletion of neutrophils in the peripheral blood within 24 hours. Differential peripheral blood neutrophil count was $77 \% \pm 1.9 \%$ in vehicle-treated mice and $0.8 \% \pm 0.3 \%$ in mice treated with the $\mathrm{mAb}$ RB6-8C5 $(P<0.0001$ vs. untreated, $n=12)$.

Ischemic ARF in neutrophil-depleted mice. Ischemic ARF was induced in neutrophil-depleted mice. Serum creatinine $(\mathrm{mg} / \mathrm{dl})$ was $0.27 \pm 0.03$ in sham-operated control mice, $2.2 \pm 0.07$ in vehicle-treated mice with ischemic $\operatorname{ARF}(P<0.001$ vs. sham), and $1.8 \pm 0.12$ in neutrophildepleted mice with ischemic ARF $(P<0.05$ vs. wild-type ischemic ARF, $P<0.001$ vs. sham) (Figure 5a).

Both vehicle-treated mice with ischemic ARF and neutrophil-depleted mice with ischemic ARF had a high ATN score (Figure 5b). Renal histopathology demonstrating extensive tubular necrosis in the outer medulla in the absence of neutrophils is shown in Figure 3c.

Caspase-1 activity, IL-18 protein expression, and neutrophil infiltration during ischemic ARF in neutrophil-depleted mice. Caspase-1 activity was increased in whole-kidney homogenates during ischemic ARF (Figure 6a). Caspase- 1 activity in ischemic ARF was the same in neutrophil-depleted mice as in vehicle-treated mice (Figure $6 a)$. IL-18 protein was determined by the ECL assay. IL-18 protein was increased in vehicle-treated mice with ischemic ARF compared with sham-operated control mice (Figure 6b). Neutrophil-depleted mice with ARF had significantly more IL-18 than shamoperated mice and less IL-18 than vehicle-treated mice with ARF (Figure 6b).

The ECL assay detects both pro-IL-18 and active IL-18. Thus, immunoblots for the active form of IL-18 $(18 \mathrm{kDa})$ were performed in whole-kidney homogenates. There was no difference in the amount of active IL-18 protein in neutrophil-depleted versus normal kidneys with ischemic ARF, confirming a neutrophil-independent source of active IL-18 (Figure 6d).

Neutrophil infiltration (neutrophils $/ \mathrm{mm}^{2}$ ) in the outer medulla was $5 \pm 0.2$ in sham-operated mice, $273 \pm 34$ in vehicle-treated mice with ischemic ARF $(P<0.01$ vs. sham $)$, and $6 \pm 1.9$ in neutrophil-depleted mice with ischemic $\operatorname{ARF}(P<0.01$ vs. ischemic ARF, NS vs. sham) (Figure 6c). Thus neutrophil depletion, like OPH-001, prevented the increase in neutrophil infiltration in the kidney during ischemic ARF.

IL-18-neutralizing antiserum protects neutrophil-depleted mice against ischemic ARF. To investigate neutrophilindependent mechanisms of IL-18-mediated injury, neutrophil-depleted mice were treated with IL-18neutralizing antiserum. Neutrophil-depleted mice with ischemic ARF treated with IL-18-neutralizing

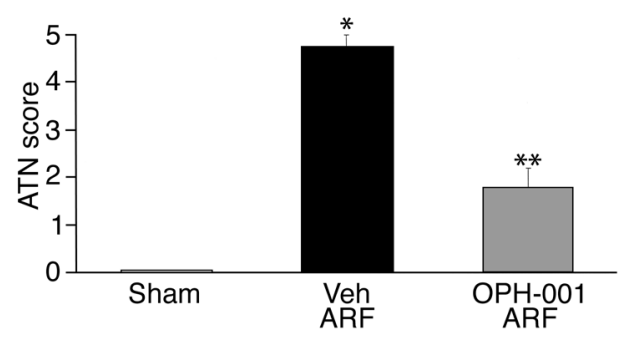

\section{Figure 2}

Mice treated with $\mathrm{OPH}-001$ are histologically protected against ischemic ARF. Mice treated with OPH-001 before induction of ischemia had a significant reduction in ATN score compared with vehicle-treated mice. ${ }^{*} P<0.001 \mathrm{vs}$. sham; ${ }^{*} P<0.001$ vs. vehicletreated ARF; $n=6$ 

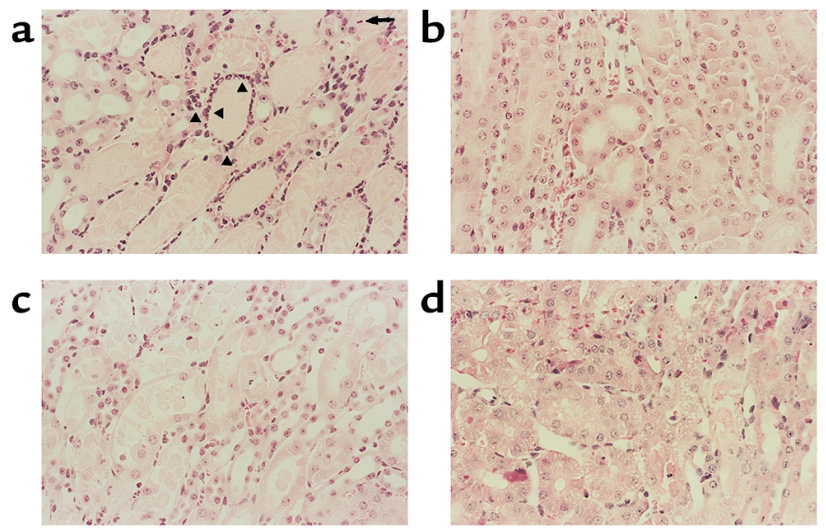

Figure 3

Renal histopathology (comparable sections and representative pictures of at least three experiments). (a) In vehicle-treated mice with ischemic ARF, proximal tubules in the outer stripe of the outer medulla show extensive damage, with epithelial cell necrosis and sloughing with focal denudation. There are occasional apoptotic cells (arrow). There are numerous clumps of neutrophils in the interstitium surrounding necrotic tubules (arrowheads). (b) In $\mathrm{OPH}-001$-treated mice with ischemic ARF, tubules are largely intact, with only focal sloughing of tubular cytoplasm and minimal loss of brush border. Neutrophils are inconspicuous. (c) In neutrophildepleted mice with ischemic ARF, proximal tubules in the outer stripe of the outer medulla show extensive damage, with epithelial cell necrosis and sloughing. Neutrophils are inconspicuous, yet there is still extensive tubular necrosis. (d) In neutrophil-depleted mice treated with IL-18-neutralizing antiserum, there is less tubular necrosis. Tubules are largely intact, with only focal sloughing of tubular cytoplasm. Neutrophils are inconspicuous.

antiserum had a $75 \%$ reduction in serum creatinine at 24 hours of reperfusion compared with vehicletreated neutrophil-depleted mice with ARF (Figure 7a). Histological scoring of ATN in the outer medulla was significantly less in neutrophil-depleted mice with ischemic ARF that were treated with IL-18neutralizing antiserum compared with vehicle-treated neutrophil-depleted mice with ischemic ARF (Figure 7b). Representative renal histopathology is shown in Figure 3d.
In additional experiments, serum creatinine was determined at 48 hours of reperfusion. Mice treated with IL-18-neutralizing antiserum before renal pedicle clamp release as well as mice treated with an additional dose of $300 \mu \mathrm{l}$ intraperitoneally at 24 hours of reperfusion were not functionally protected against ischemic ARF at 48 hours of reperfusion compared with vehicle-treated mice with ischemic ARF.

IL-18 and proximal tubules. We have previously demonstrated an increase in IL-18 in the urine of mice with ischemic ARF (7). Thus, we investigated proximal tubules as the possible source and target of IL-18. Studies were performed on freshly isolated proximal tubules from $\mathrm{C} 57 \mathrm{BL} / 6$ mice. OPH-001 protected against 25 minutes of hypoxic injury in these tubules. LDH release was $11 \% \pm 1 \%$ in normoxic tubules, $38 \% \pm 6 \%$ in hypoxic tubules preincubated with vehicle (DMSO) $(P<0.001$ vs. normoxia, $n=6)$, and $22 \% \pm 1 \%$ in hypoxic tubules preincubated with OPH-001 $(100 \mu \mathrm{M})(P<0.01$ vs. hypoxia, $n=6)$. Immunoblotting $(n=6)$ of normoxic proximal tubules demonstrated the presence of pro-IL-18 (24 $\mathrm{kDa})$. Exogenous recombinant IL-18 (1 $\mu \mathrm{g}$ per $6 \mathrm{ml}$ of tubule suspension) exacerbated sublethal (12 minutes) hypoxic proximal tubular injury. LDH release was $10 \% \pm 1 \%$ in normoxic tubules, $13 \% \pm 1 \%$ in hypoxic tubules preincubated with vehicle (saline) (NS vs. normoxia, $n=6$ ), and $18 \% \pm 1 \%$ in hypoxic tubules preincubated with recombinant IL-18 before induction of hypoxia $(P<0.01$ vs. hypoxia, $n=6)$. It will be interesting to further study the role of IL-18 in hypoxic injury in proximal tubules.

$O P H-001$ inhibits both caspase- 1 and caspase- 3 . The $\mathrm{IC}_{50}$ of OPH-001 for recombinant caspase- 1 was determined to be $6.5 \mu \mathrm{M}$. The $\mathrm{IC}_{50}$ of OPH-001 for recombinant caspase- 3 was determined to be $1.0 \mu \mathrm{M}$. Thus OPH-001 is a caspase inhibitor that inhibits both caspase- 1 and caspase-3 in vitro.

To confirm that OPH-001 also inhibits proapoptotic caspase- 3 in vivo, both caspase- 3 activity and apoptosis were determined. Caspase- 3 activity was increased during ischemic ARF. OPH-001 inhibited
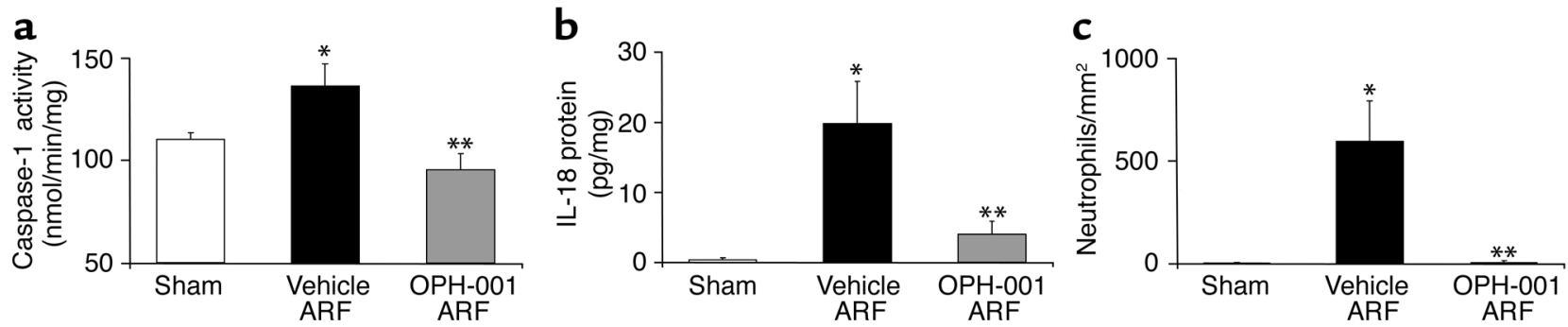

\section{Figure 4}

Mice treated with OPH-001 have decreased caspase-1 activity (a), IL-18 protein (b), and neutrophil infiltration (c) in the kidney in mice with ischemic ARF. (a) OPH-001 resulted in a normalization of caspase- 1 activity compared with that of vehicle-treated mice. ${ }^{*} P<0.05$ vs. sham; ${ }^{*} P<0.05$ vs. vehicle-treated ARF, NS vs. sham; $n=4$. (b) IL-18 protein (ECL assay) was increased in ischemic ARF. OPH-001 resulted in a normalization of IL-18 compared with that of vehicle-treated mice. ${ }^{*} P<0.01$ vs. sham; ${ }^{*} P<0.01$ vs. vehicle-treated ARF, NS vs. sham; $n=4$. (c) Neutrophil infiltration (neutrophils $/ \mathrm{mm}^{2}$ ) was increased in vehicle-treated mice with ischemic ARF and prevented in OPH-001-treated mice with ischemic ARF. ${ }^{*} P<0.01$ vs. sham; ${ }^{*} P<0.01$ vs. vehicle-treated ARF, NS vs. sham; $n=5$. 


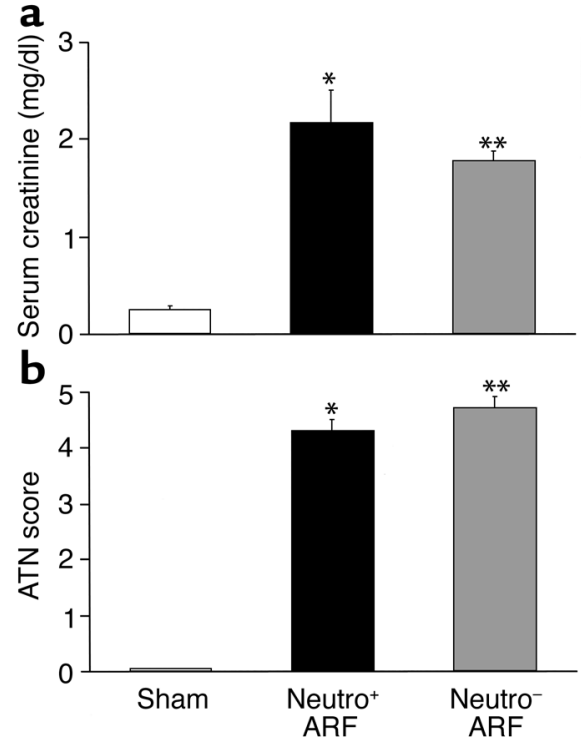

the increase in caspase- 3 activity. Caspase- 3 activity $(\mathrm{nmol} / \mathrm{min} / \mathrm{mg}$ ) was $9.6 \pm 1.7$ in sham-operated control mice, $31.2 \pm 4.6$ in vehicle-treated mice with ischemic $\operatorname{ARF}(P<0.01$ vs. sham, $n=4)$, and $16.5 \pm 4.2$ in OPH-001-treated mice with ischemic ARF $(P<0.01$ vs. vehicle-treated ischemic ARF, NS vs. sham, $n=4)$. The number of apoptotic tubular epithelial cells in the outer stripe of the outer medulla per ten highpower fields was 0 in sham-operated control mice, $10.6 \pm 3.6$ in vehicle-treated mice with ischemic ARF $(P<0.05$ vs. sham, $n=4)$, and $2.7 \pm 2.1$ in OPH-001treated mice with ischemic ARF $(P<0.05$ vs. vehicletreated ischemic ARF, $n=6$ ).

Specificity of OPH-001. We have determined the specificity of OPH-001 for caspases versus two other major cysteine proteases, calpain and cathepsin B, both of which are mediators of cell death. The $\mathrm{IC}_{50}$ of OPH-001 for purified calpain was $727 \mu \mathrm{M}$. The $\mathrm{IC}_{50}$ for purified cathepsin B was $218 \mu \mathrm{M}$. These values are

\section{Figure 6}

Caspase-1 activity (a), IL-18 protein (b), neutrophil infiltration (c), and active form of IL-18 (d) in the kidney in neutrophil-depleted mice with ischemic ARF. (a) Caspase-1 activity increased in both vehicletreated $\left(\right.$ neutro $\left.^{+}\right)$and neutrophil-depleted (neutro ${ }^{-}$) mice with ARF. ${ }^{*} P<0.001$ vs. sham; ${ }^{*} P<0.001$ vs. sham, NS vs. neutro ${ }^{+} ; n=5$. (b) IL-18 protein was measured by the ECL assay that detects both pro-IL-18 and active IL-18. IL-18 was increased in ischemic ARF in neutro $^{+}$as well as neutro- mice with ischemic ARF compared with sham-operated controls. IL-18 was higher in neutro ${ }^{+}$mice than in neutro- mice. ${ }^{*} P<0.001$ vs. sham; ${ }^{*} P<0.01$ vs. sham, $P<0.01$ vs. neutro ${ }^{+} ; n=11$. (c) Neutrophil infiltration (neutrophils $/ \mathrm{mm}^{2}$ ) was increased in ischemic ARF in neutro ${ }^{+}$and prevented in neutro- mice with ischemic ARF. ${ }^{*} P<0.01$ vs. sham; ${ }^{*} P<0.01$ vs. vehicle-treated ARF, NS vs. sham; $n=7$. (d) There was no difference in the amount of active IL-18 protein $(18 \mathrm{kDa})$ on immunoblot analysis in whole-kidney homogenates in neutro ${ }^{+}$versus neutro- mice with ischemic ARF. Recombinant murine IL-18 (PeproTech Inc., Rocky Hill, New Jersey, USA) was used as a positive control (Pos). A representative picture of three separate experiments is shown.

\section{Figure 5}

Neutrophil-depleted mice have slight functional protection against ischemic $\operatorname{ARF}$ (a) and are not histologically protected against ischemic ARF (b). (a) There was an increase in serum creatinine in vehicle-treated mice with ischemic ARF $\left(\right.$ neutro $\left.{ }^{+}\right)$. Neutrophildepleted mice with ischemic ARF (neutro-) had a slight decrease in serum creatinine compared with neutro ${ }^{+}$mice. ${ }^{*} P<0.001 \mathrm{vs}$. sham; ${ }^{*} P<0.05$ vs. neutro ${ }^{+}$ARF, $P<0.001$ vs. sham; $n=8$. (b) Histological scoring of ATN in the outer medulla was the same in neutro ${ }^{+}$ as in neutro- mice. ${ }^{*} P<0.001$ vs. sham; ${ }^{*} P<0.001$ vs. sham, NS vs. neutro $; n=7$. The lack of protection occurs despite the prevention of neutrophil infiltration in the kidney in mice with ischemic ARF, shown in Figure $6 c$.

considerably higher than the $\mathrm{IC}_{50}$ for caspase- 1 and caspase- 3 reported above. We also measured cathepsin B activity in the kidney during ischemic ARF. There was no increase in cathepsin B activity in the kidney in mice with ischemic ARF compared with sham-operated mice, and OPH-001 had no effect on cathepsin B activity. Cathepsin B activity $(\mu \mathrm{mol} / \mathrm{min} / \mathrm{mg})$ was $51.2 \pm 6$ in sham-operated control mice, $52 \pm 2$ in vehicle-treated mice with ischemic ARF (NS vs. sham, $n=4$ ), and $53.6 \pm 3$ in OPH-001treated mice with ischemic ARF (NS vs. vehicle-treated ischemic ARF, $n=4$ ).

\section{Discussion}

The caspases are a family of intracellular cysteine proteases that have a cysteine residue at their active site. Caspase-1 plays a major role in the activation of the proinflammatory cytokines IL- $1 \beta$ and IL-18 (6). Caspase-3 is the "executioner caspase" that is centrally important in apoptotic cell death in vivo. In vivo administration of a caspase inhibitor affords

a

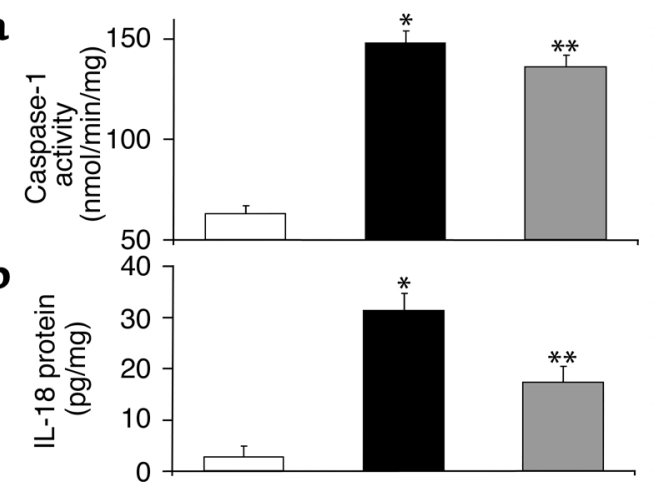

c

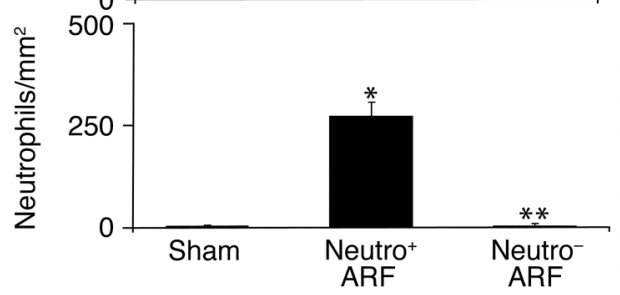

d

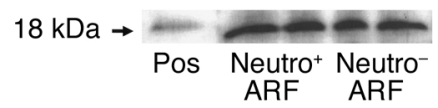



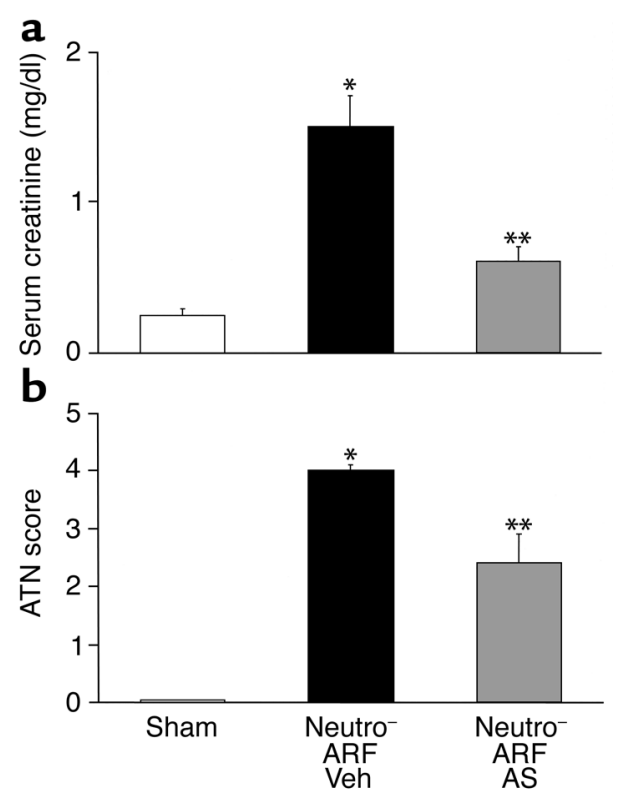

Figure 7

Neutrophil-depleted mice treated with IL-18-neutralizing antiserum are both functionally (a) and histologically (b) protected against ischemic ARF. Mice were injected with the neutrophil-depleting antibody RB6-8C5 24 hours before renal pedicle clamp (neutro-), followed by anti-IL-18 antiserum (AS) or vehicle 40 minutes before renal pedicle clamp and just before clamp release. (a) In vehicletreated neutro- mice with ischemic ARF, there was a significant increase in serum creatinine compared with sham-operated controls. In neutro- mice treated with AS, the serum creatinine was significantly decreased compared with vehicle-treated mice with ARF. ${ }^{*} P<0.01$ vs. sham; ${ }^{*} P<0.01$ vs. vehicle-treated ARF, NS vs. sham; $n=8$. (b) In vehicle-treated neutro- mice with ischemic ARF, there was a significant increase in ATN score compared with sham-operated controls. In neutro- mice treated with AS before induction of ischemic ARF, the ATN score was significantly decreased compared with vehicle-treated neutro- mice with ARF. ${ }^{*} P<0.001$ vs. sham; ${ }^{*} P<0.01$ vs. vehicle-treated ARF; $n=4$.

protection against apoptotic cell injury in animal models of hypoxic-ischemic brain injury (13) and myocardial reperfusion injury after coronary artery occlusion (14). A novel caspase inhibitor, IDN-1965, reduces endothelial cell apoptosis and prolongs animal survival after orthotopic liver transplantation in vivo (15). Thus the role of caspases in organ injury due to apoptosis in brain, heart, and liver is well established. However, the predominant morphological change in the proximal tubule in animal models of ischemic ARF and in posttransplant ARF in humans is necrosis; hence the term "acute tubular necrosis" (ATN) (16). Based on findings that caspases are mediators of in vitro hypoxic proximal tubular necrosis $(1,2)$ and that caspase inhibition has a protective effect on ischemic injury in other organs, we investigated the effect of caspase inhibition in ischemic ARF. In the present study, we demonstrate that the newly developed caspase inhibitor OPH-001 has a remarkable protective effect on function and ATN in ischemic ARF in mice. Thus, in contrast to caspase-mediated apoptosis in ischemic organ injury in brain, heart, and liver, we describe the role of caspases in necrotic cell injury in the kidney in vivo.

We have previously demonstrated that impaired IL-18 processing protects caspase-1-deficient mice from ischemic ARF (7). In this study, IL-18-neutralizing antiserum resulted in a degree of protection from ischemic ARF similar to that seen in caspase-1-deficient mice. Because the caspase-1-deficient mice showed decreased neutrophil infiltration in ischemic ARF, we hypothesized that the deleterious role of IL-18 may be due to increased neutrophil infiltration. Therefore, in the present study, we determined the effect of caspase inhibition on caspase- 1 activity, IL-18 protein, and neutrophil infiltration in the kidney in ischemic ARF. OPH-001 significantly reduced the increase in caspase- 1 activity and IL-18 and prevented neutrophil infiltration in the kidney during ischemic ARF.

There is evidence that leukocytes, particularly neutrophils, mediate tubular injury in ARF and play a key role in the development of acute renal failure (17). This evidence is derived from studies that show an accumulation of neutrophils in ischemic ARF and studies demonstrating a beneficial role of anti-ICAM-1 therapy in ARF (18). In addition, mice depleted of peripheral neutrophils by antineutrophil serum were protected against ischemic ARF (18). However, in another study, rats depleted of peripheral neutrophils by antineutrophil serum were not protected against ischemic ARF (19). In both studies, quantitation of neutrophils in the kidney during ischemic ARF was not reported. In the present study, the slight functional protection and lack of histological protection against ischemic ARF in neutrophil-depleted mice argue against a significant role of neutrophils in the C57BL/6 mouse model of ischemic ARF.

Other leukocytes, e.g., lymphocytes and monocytes, have recently been studied in ischemic ARF. The role of lymphocytes in ischemic ARF is controversial. In one study, CD4 T lymphocyte-deficient mice were protected against ischemic ARF (20). However, in another study, mice deficient in recombination-activating gene-1 (RAG-1), which have no mature T or B lymphocytes, were not protected against ischemic ARF (21). Ischemic ARF elicits an inflammatory response that may also involve macrophages as evidenced by macrophage infiltration in the postischemic kidney (20). Blocking the costimulatory pathway with a monoclonal anti-B7-1 antibody is associated with protection against ischemic $\mathrm{ARF}$ and less monocyte adherence in the vasa recta (22). As T lymphocytes and monocytes are a known source of IL-18, it is possible that these cells are a source of IL-18 in ischemic ARF. Another possible source and target of IL18 in ischemic ARF is the proximal tubule itself. In the present article, we present novel data regarding the proximal tubule as a possible source and target of IL-18.

IL-18 is a mediator of inflammation and tissue injury in many organs. Protection against experimental colitis in caspase-1-deficient mice is associated 
with reduced release of IL-18 from the colon (23). Neutralization of IL-18 during lethal endotoxemia reduces neutrophil tissue accumulation and protects mice against the lethal effects of LPS (24). Neutrophil activation by IL-18 in vivo has been described (25). The use of IL-18-neutralizing antiserum has demonstrated the important role of IL-18 in mediating inflammation in models of arthritis (26), lung injury (27), and inflammatory bowel disease (28). Thus, we investigated the role of IL-18-mediated neutrophil infiltration in the kidney in ischemic ARF.

Both the caspase inhibitor OPH-001 and the neutrophil-depleting antibody RB6-8C5 prevented neutrophil infiltration in the kidney in ischemic ARF. The lack of protection against ischemic ARF in neutrophildepleted mice despite the increase in caspase- 1 activity and IL-18 protein suggested neutrophil-independent mechanisms of IL-18-mediated renal injury. IL-18-neutralizing antiserum protected against ischemic ARF in neutrophil-depleted mice, demonstrating a neutrophil-independent mechanism of IL-18-mediated proximal tubular injury. The degree of protection of renal function is similar to what we have previously reported in wild-type mice (7). These data challenge the concept that IL-18-mediated organ injury is mediated by neutrophil-dependent inflammation as demonstrated in lethal endotoxemia (24), experimental colitis in mice (23), rheumatoid arthritis $(26,29)$, acute lung injury $(27,30)$, and inflammatory CNS diseases (31).

We demonstrate that OPH-001 also inhibits caspase- 3 activity and apoptosis during ischemic ARF. In the present study, the beneficial effects of OPH-001 are attributed to a decrease in necrosis rather than to a decrease in apoptosis for the following reasons. (a) Apoptosis was seen only in a small minority of tubular cells (less than $1 \%$ of the tubules; Figure 3a). Necrosis was seen in $46-75 \%$ of tubular cells (Figure 3a). (b) In ischemic ARF in animal models and delayed renal graft function in humans, necrosis of proximal tubules is the predominant finding (32). Inhibition of this necrosis by a wide variety of agents results in functional protection against ARF (33). (c) In other studies of ischemic ARF, there was no increase in the number of apoptotic cells at 24 and 48 hours of reperfusion despite extensive tubular necrosis and renal failure $(9,34)$. The relative contribution of the small amount of apoptosis to ischemic ARF is not known (35). Thus, it is unlikely that the extensive functional and histological protection against tubular necrosis induced by OPH-001 is due to the effects of slight apoptosis inhibition.

A recent study demonstrated that caspase inhibition in ischemic ARF in mice was associated with decreased apoptosis and inflammation (5). However, in this study, there was a lack of specificity in the means used to assess apoptosis, acute tubular necrosis was not quantitated, and proinflammatory caspase- 1 was not considered. The upstream pathways of apoptosis and necrosis in ischemic ARF in the kidney may be similar, as evidenced by studies that endonuclease activation, considered to be a feature of apoptosis, also occurs in necrotic proximal tubular cells without morphological features of apoptosis (36). While OPH-001 did decrease apoptosis, its predominant effect was on proximal tubular necrosis.

In conclusion, this study is, to our knowledge, the first demonstration of the protective effect of a caspase inhibitor against ATN and associated functional ARF. This protective effect of caspase inhibition is associated with decreased caspase-1 activity, decreased IL-18 protein, and prevention of neutrophil infiltration in the kidney. Remarkably, the use of neutrophil-depleted mice failed to demonstrate a significant role of neutrophils in our C57BL/6 model of ischemic ARF. Rather, a neutrophil-independent effect of proinflammatory IL-18 was demonstrated in studies in which IL-18-neutralizing antiserum gave significant protection in neutrophil-depleted mice. The potential therapeutic effect of caspase inhibition may have future clinical implications for human ARF, which still has a mortality of over $50 \%$.

\section{Acknowledgments}

This work was supported by NIH grant 1 RO1 DK56851 (to C.L. Edelstein). D. Ljubanovic was an International Society of Nephrology Fellow in renal pathology. We thank Charles A. Dinarello for his helpful comments and for providing the IL-18-neutralizing antiserum.

1. Kaushal, G.P., Ueda, N., and Shah, S.V. 1997. Role of caspases (ICE/CED 3 proteases) in DNA damage and cell death in response to a mitochondrial inhibitor, antimycin A. Kidney Int. 52:438-445.

2. Edelstein, C.L., Shi, Y., and Schrier, R.W. 1999. Role of caspases in hypoxia-induced necrosis of rat renal proximal tubules. J. Am. Soc. Nephrol. 10:1940-1949.

3. Kaushal, G.P., Singh, A.B., and Shah, S.V. 1998. Identification of gene family of caspases in rat kidney and altered expression in ischemia reperfusion injury. Am. J. Physiol. 274:F587-F595.

4. Shi, Y., Melnikov, V.Y., Schrier, R.W., and Edelstein, C.L. 2000. Downregulation of the calpain inhibitor protein, calpastatin, by caspases during renal ischemia. Am. J. Physiol. 279:F509-F517.

5. Daemen, M.A.R.C., et al. 1999. Inhibition of apoptosis induced by ischemia-reperfusion prevents inflammation. J. Clin. Invest. 104:541-549.

6. Fantuzzi, G., Puren, A.J., Harding, M.W., Livingston, D.J., and Dinarello, C.A. 1998. Interleukin-18 regulation of interferon gamma production and cell proliferation as shown in interleukin-1 beta-converting enzyme (caspase-1)-deficient mice. Blood. 91:2118-2125.

7. Melnikov, V.Y., et al. 2001. Impaired IL-18 processing protects caspase1-deficient mice from ischemic acute renal failure. J. Clin. Invest. 107:1145-1152.

8. Wipke, B.T., and Allen, P.M. 2001. Essential role of neutrophils in the initiation and progression of a murine model of rheumatoid arthritis. J. Immunol. 167:1601-1608.

9. Gobe, G., et al. 2000. Relationship between expression of Bcl-2 genes and growth factors in ischemic acute renal failure in the rat. J. Am. Soc. Nephrol. 11:454-467.

10. Fantuzzi, G., Reed, D.A., and Dinarello, C.A. 1999. IL-12-induced IFNgamma is dependent on caspase-1 processing of the IL- 18 precursor. J. Clin. Invest. 104:761-767.

11. Hillegass, L.M., Griswold, D.E., Brickson, B., and Albrightson-Winslow, C. 1990. Assessment of myeloperoxidase activity in whole rat kidney. J. Pharmacol. Methods. 24:285-295.

12. Ysebaert, D.K., et al. 2000. Identification and kinetics of leukocytes after severe ischaemia/reperfusion renal injury. Nephrol. Dial. Transplant. 15:1562-1574. 
13. Cheng, Y., et al. 1998. Caspase inhibitor affords neuroprotection with delayed administration in a rat model of neonatal hypoxic-ischemic brain injury. J. Clin. Invest. 101:1992-1999.

14. Yaoita, H., Ogawa, K., Maehara, K., and Maruyama, Y. 1998. Attenuation of ischemia/reperfusion injury in rats by a caspase inhibitor. Circulation. 97:276-281.

15. Natori, S., et al. 1999. Apoptosis of sinusoidal endothelial cells occurs during liver preservation injury by a caspase-dependent mechanism. Transplantation. 68:89-96.

16. Lieberthal, W., and Nigam, S.K. 2000. Acute renal failure. II. Experimental models of acute renal failure: imperfect but indispensable. Am. J. Physiol. 278:F1-F12.

17. Heinzelmann, M., Mercer-Jones, M.A., and Passmore, J.C. 1999. Neutrophils and renal failure. Am. J. Kidney Dis. 34:384-399.

18. Kelly, K.J., Williams, W.W., Jr., Colvin, R.B., and Bonventre, J.V. 1996. Intracellular adhesion molecule-1 deficient mice are protected against ischemic renal injury. J. Clin. Invest. 97:1056-1063.

19. Paller, M.S. 1989. Effect of neutrophil depletion on ischemic renal injury in the rat. J. Lab. Clin. Med. 113:379-386.

20. Burne, M.J., et al. 2001. Identification of the $\mathrm{CD}^{+} \mathrm{T}$ cell as a major pathogenic factor in ischemic acute renal failure. J. Clin. Invest. 108:1283-1290. doi:10.1172/JCI200112080.

21. Park, P., et al. 2002. Injury in renal ischemia-reperfusion is independent from immunoglobulins and T lymphocytes. Am. J. Physiol. Renal Physiol. 282:F352-F357.

22. De Greef, K.E., et al. 2001. Anti-B7-1 blocks mononuclear cell adherence in vasa recta after ischemia. Kidney Int. 60:1415-1427.

23. Siegmund, B., Lehr, H.A., Fantuzzi, G., and Dinarello, C.A. 2001. IL-1 beta-converting enzyme (caspase-1) in intestinal inflammation. Proc Natl. Acad. Sci. USA. 98:13249-13254.

24. Netea, M.G., et al. 2000. Neutralization of IL-18 reduces neutrophil tissue accumulation and protects mice against lethal Escherichia coli and Salmonella typhimurium endotoxemia. J. Immunol. 164:2644-2649.
25. Leung, B.P., et al. 2001. A role for IL-18 in neutrophil activation. J. Immunol. 167:2879-2886.

26. Plater-Zyberk, C., et al. 2001. Therapeutic effect of neutralizing endogenous IL-18 activity in the collagen-induced model of arthritis. J. Clin Invest. 108:1825-1832. doi:10.1172/JCI200112097.

27. Jordan, J.A., et al. 2001. Role of IL-18 in acute lung inflammation. J. Immunol. 167:7060-7068.

28. Siegmund, B., et al. 2001. Neutralization of interleukin-18 reduces severity in murine colitis and intestinal IFN-gamma and TNF-alpha production. Am.J. Physiol. Regul. Integr. Comp. Physiol. 281:R1264-R1273.

29. Gracie, J.A., et al. 1999. A proinflammatory role for IL-18 in rheumatoid arthritis. J. Clin. Invest. 104:1393-1401.

30. Arndt, P.G., Fantuzzi, G., and Abraham, E. 2000. Expression of interleukin-18 in the lung after endotoxemia or hemorrhage-induced acute lung injury. Am. J. Respir. Cell Mol. Biol. 22:708-713.

31. Fassbender, K., et al. 1999. Interferon-gamma-inducing factor (IL-18) and interferon-gamma in inflammatory CNS diseases. Neurology. 53:1104-1106.

32. Lieberthal, W., and Nigam, S.K. 1998. Acute renal failure. Relative importance of proximal vs. distal tubular injury. Am. J. Physiol. 275:F623-F632.

33. Edelstein, C.L., and Schrier, R.W. 2001. Pathophysiology of ischemic acute renal failure. In Diseases of the kidney and urinary tract. R.W. Schrier, editor. Lippincott Williams \& Wilkins. Philadelphia, Pennsylvania, USA. 1041-1069.

34. Lieberthal, W., et al. 2001. Rapamycin impairs recovery from acute renal failure: role of cell-cycle arrest and apoptosis of tubular cells. Am. J. Physiol. Renal Physiol. 281:F693-F706.

35. Bonegio, R., and Lieberthal, W. 2002. Role of apoptosis in the pathogenesis of acute renal failure. Curr. Opin. Nephrol. Hypertens. 11:301-308.

36. Ueda, N., Walker, P.D., Hsu, S., and Shah, S.V. 1995. Activation of a 15$\mathrm{kDa}$ endonuclease in hypoxia/reoxygenation injury without morphologic features of apoptosis. Proc. Natl. Acad. Sci. USA. 92:7202-7206. 